Chirurg 2019 90 (Suppl 2):S62 https://doi.org/10.1007/s00104-019-0899-z Online publiziert: 13. Februar 2019 (c) Springer Medizin Verlag GmbH, ein Teil von Springer Nature 2019

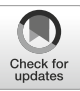

M. Schrempf $\cdot$ M. Anthuber

Klinik für Allgemein-, Viszeral- Transplantationschirurgie, Klinikum Augsburg, Augsburg, Deutschland

\title{
Einfluss des tumorfreien Resektionsrandes auf das Gesamt- und krankheitsfreie Überleben nach Resektion kolorektaler Lebermetastasen
}

Beide Definitionen wurden in der statistischen Auswertung berücksichtigt.

Margonis GA, Sergentanis TN, NtanasisStathopoulos I et al (2018) Impact of surgical margin width on recurrence and overall survival following $\mathrm{R} 0$ hepatic resection of colorectal metastases. Ann Surg 267:1047-1055

Hintergrund. Die Resektion von Lebermetastasen (LM) des kolorektalen Karzinoms (KRK) stellt im Gesamtkonzept einer multimodalen Therapie die wesentliche Behandlungsoption in Bezug auf Heilung dar. Es ist allgemein anerkannt, dass sich eine R0-Resektion positiv auf die Prognose auswirkt. Die optimale Größe des tumorfreien Resektionsrandes ist zwar seit Jahrzehnten Gegenstand der chirurgischen Forschung, jedoch weiterhin umstritten.

Methoden. Die vorliegende Metaanalyse untersuchte die Assoziation zwischen Größe des tumorfreien Resektionsrandes und Gesamtüberleben („overall survival“, OS) sowie krankheitsfreiem Überleben („disease free survival“, DFS). In den eingeschlossenen Studien wurden zwei verschiedene Definitionen des R1-Status verwendet. R1 wurde entweder definiert als das Vorliegen von Tumorzellen weniger als $1 \mathrm{~mm}$ vom Resektionsrand entfernt oder als das Vorliegen von Tumorzellen am Resektionsrand $(0 \mathrm{~mm})$.

Dieser Beitrag wurde erstpubliziert in Der Chirurg (2018) 89:733-733. https://doi.org/10. 1007/s00104-018-0687-1
Ergebnisse. Es wurden 34 Studien mit tion in die Metaanalyse eingeschlossen. Unter allen R0-Resektionen war ein größerer tumorfreier Resektionsrand $(>1 \mathrm{~cm}$ vs. $<1 \mathrm{~cm})$ mit einem verbesserten $3-$, 5- und 10-Jahres-OS assoziiert (3 Jahre: gepooltes relatives Risiko $[R R]=0,86$, $95 \%$-Konfidenzintervall [KI] 0,79-0,95; 5 Jahre: gepooltes $\mathrm{RR}=0,91,95 \%$-KI 0,85-0,97; 10 Jahre: gepooltes $\mathrm{RR}=0,94$, $95 \%$-KI 0,88-1,00). Ebenso war für R0Resektionen mit größerem tumorfreiem Resektionsrand ( $>1 \mathrm{~cm}$ vs. $<1 \mathrm{~cm}$ ) eine Assoziation mit einem verbesserten 3-, 5- und 10-Jahres-DFS nachweisbar. Für 5 Patientenkollektive waren Daten zur R1-Situation für beide R1-Definitionen vorhanden. Ein tumorfreier Resektionsrand $>1 \mathrm{~cm}$ war sowohl im Vergleich mit der Gruppe $0-10 \mathrm{~mm}$ als auch im Vergleich mit der Gruppe $1-10 \mathrm{~mm}$ mit einem verbesserten 5-Jahres-OS assoziiert (>1 cm vs. $0-10 \mathrm{~mm}$ : RR 0,92 $95 \%$-KI $0,87-0,97$; $>1 \mathrm{~cm}$ vs. $1-10 \mathrm{~mm}$ : RR 0,94, $95 \%$-KI 0,88-0,99). Tumorfreie Resektionsränder $>1 \mathrm{~mm}$ waren im Vergleich zum tumorfreien Resektionsrändern $<1 \mathrm{~mm}$ für alle untersuchten Zeiträume mit einem verbesserten OS assoziiert (3 Jahre: gepooltes $\mathrm{RR}=0,77$, 95\%-KI 0,63-0,93; 5 Jahre: gepooltes $\mathrm{RR}=0,80$, $95 \%$-KI 0,72-0,88; 10 Jahre: gepooltes $\mathrm{RR}=0,95,95 \%$-KI 0,92-0,99). In der Metaregressionsanalyse ergab sich kein Hinweis für einen Einfluss von Geschlecht, Alter, dem Vorliegen syneiner Gesamtzahl von 11.147 Leberresek- chroner Metastasen, neoadjuvanter oder adjuvanter Behandlung, Lokalisation des Primarius oder Anzahl der LM.

Diskussion und Fazit. Die vorliegende Metaanalyse zeigt, dass R0-Resektionen mit einem Abstand $>1 \mathrm{~cm}$ im Vergleich $\mathrm{zu}$ R0-Resektionen mit einem Abstand $<1 \mathrm{~cm}$ mit einem verbesserten OS und DFS assoziiert sind. Tumorfreie Resektionsränder im Submillimeterbereich wiesen ein schlechteres onkologisches Outcome auf als Resektionsränder $>1 \mathrm{~mm}$. Dhir et al. zeigten bereits 2011 in einer Metaanalyse, dass ein tumorfreier Resektionsrand $>1 \mathrm{~cm}$ mit einer verbesserten Prognose assoziiert ist [1]. Diese Ergebnisse wurden später allerdings infrage gestellt, da Dhir et al. u. a. nicht die Gabe einer (neo)adjuvanten Chemotherapie berücksichtigt hatten. In der vorliegenden Arbeit war die Assoziation zwischen größerem Resektionsrand und verbessertem OS und DFS unabhängig von der Gabe einer (neo)adjuvanten Chemotherapie.

\section{Korrespondenzadresse}

\section{Prof. Dr. M. Anthuber}

Klinik für Allgemein-, Viszeral- Transplantationschirurgie, Klinikum Augsburg Stenglinstr. 2, 86156 Augsburg, Deutschland matthias.anthuber@klinikum-augsburg.de

Interessenkonflikt. M. Schrempf und M. Anthuber geben an, dass kein Interessenkonflikt besteht.

\section{Literatur}

1. Dhir M, Lyden ER, Wang A et al (2011) Influence of margins on overall survival after hepatic resection for colorectal metastasis. Ann Surg 254:234-242 